\title{
NEW DESIGN METHODOLOGY - USING VHDL-AMS MODELS TO CONSIDER AGING EFFECTS IN AUTOMOTIVE MECHATRONIC CIRCUITS FOR SAFETY RELEVANT FUNCTIONS'
}

\author{
Prof. emer. Georg Scharfenberg \\ Faculty of Electrical Engineering and Information \\ Technology \\ OTH Regensburg \\ Regensburg 93053, Germany
}

\begin{abstract}
In this paper a design method for mechatronic functions in an early design phase under consideration of the requirements of functional safety is proposed [14]. The goal of the method is to discover threats for the safety goals and to prove or optimize the diagnostic mechanisms. At the current design process a lifetime endurance test is performed in a late project phase. In order to avoid unexpected late design changes the method should be applied in an early design phase. The method is an additional way to avoid design failures for $E / E$ functions with special focus on aging in the field of functional safety applications. The method is developed out of three standards VDI 2221 methodology for development and construction of technical systems and products [1]), VDI 2206 design methodology for mechatronic systems [2] and the ISO 26262 road vehicles - Functional Safety [3] by using the V-Model. Furthermore, a core element of the design method is simulating aging effects by applying VHDL - AMS. To demonstrate the design methodology a sensor out of the EGAS function is chosen as a safety relevant automotive system. The limitation is the availability of aging data for electrical components.
\end{abstract}

Keywords-Functional Safety; Mechatronic design metho; Resistor aging

\section{INTRODUCTION}

The automotive industry encounters a transformation from a mechanical dominated industry to a stronger electronic and information influenced technology. Technical changes caused by e-mobility, connectivity or autonomous driving require more and more electric / electronic (E/E). Studies show that up to $90 \%$ of the innovations in the automotive sector are only possible through the use of electronics and software [compare 4].

As a rule, the new electronic systems must fulfil safety-relevant requirements which are controlled by electronics or software and have to follow the international standard ISO 26262 [3].

Circuits in the automotive industry are designed in a way that they remain functional in all conditions

\author{
Ludek Elis, Gerhard Hofmann \\ KAE - Department of Applied Electronics and \\ Telecommunications, \\ University of West Bohemia, Univerzitni 8, \\ 30614 Pilsen, Czech Republic
}

despite physical stress over lifetime. To ensure this, in the automotive industry, an environmental validation with real sample parts in the development phase serves as state of the art (for example see ISO 16750$4: 2010$ [5]). The validation is normally executed in a late design phase, when stable samples are available. The idea of the new methodology is to develop a simulation-assisted way focusing on aging effects, by means of which a threat to the safety goal can be detected or ruled out. Especially, the change of electrical properties is considered via aging, and it is investigated if mechanism of diagnosis can be bypassed or generate a failure.

A worst-case circuit analysis is generally performed to evaluate the product development of the hardware design. Thereby ageing effects with limit values are statically considered. The presented method provides a deeper understanding of ageing effects over time. With the availability of the aging models for the safety-relevant components, the presented method additionally offers the possibility to redefine the worst-case limit value. The redefinition of the limit values can advantageously facilitate the solvability of a problematic design and lead to an improvement of the circuit tolerances.

\section{NEW DESIGN METHODOLOGY}

Out of the theoretical considerations of the requirements of the standards VDI 2221 "methodology for development and construction of technical systems and products" [1], VDI 2206 "design methodology for mechatronic systems" [2] and the ISO 26262 "road vehicles - functional safety" processes of functional safety [3] the new methodology is developed with special focus on aging.

\section{A. General development methodology according to VDI 2221}

The VDI 2221 methodology for development and construction of technical systems and products [1] is a general standard for design and can be used for all engineering disciplines. The standard proposes to divide a problem solution in four serial phases: 
a) Planning phase;

b) Conceptual phase;

c) Design phase;

d) Elaboration phase.( compare [1] page 9)

The further a project goes, and the more details are known the phases can be repeated more often. [1]

\section{B. Mechatronic development standard according to VDI 2206}

The standard VDI 2206 is a supplement of the VDI 2221 with focus on mechatronic. Mechatronic is defined as the combination of the fields electrical engineering, mechanical engineering and computer science.

As mechatronic deals with different physical disciplines VHDL-AMS was chosen as an appropriate simulation language for different areas of physics, such as electronics, mechanics or thermodynamics, because it can handle not only electrical simulation.

The VDI 2206 standard proposes three principles for designing mechatronic:

"• general problem-solving cycle on the micro-level

- V model (...)

- predefined process modules for handling recurrent working steps in the development of mechatronic systems" ([2] page 26).

The general problem solving is like the approach of the VDI 2221 with the focus on micro level.

The V-model is known from software development (compare [6], page 70) and focuses on a macro level.

The usage of predefined process modules requests to use standardized modules. Existing simulation modules are a strong indicator that is predefined and reusable.

\section{Design and verification process requirements according to ISO 26262 (compare [3])}

The ISO 26262 covers the whole life cycle from design, production and recycling. In part 3 of the ISO26262 the item has to be specified from the beginning. Out of the hazard and risk analysis an "automotive safety integrity level (ASIL)" is assigned. After the ASIL classification a safety concept and safety goal are required. Part 4 deals with the "product development at the system level". As aging can only occur at a hardware level, part 5 "product development at the hardware level" is relevant for the new methodology.

Highly recommended for all ASIL levels for an inductive analysis is the failure mode and effect analysis (FMEA) (compare [3] part 5, page 11). So, a core component of the new methodology is a FMEA with the focus on aging - it is called "Aging FMEA"([14], page 54).

The ISO 26262 standard gives some hints to simulation - especially in chapter "hardware design verification" ([3] part 5, page 12).

Out of the remaining parts only part 8 handled supporting processes, especially the verification and qualification processes, is important due to the new design methodology. The standard in general is not explicitly focused on aging.

\section{New design methodology}

To develop the new design methodology the four phases of the VDI 2221 and the V-model of the VDI 2206 are combined. This is visualized in figure 1.

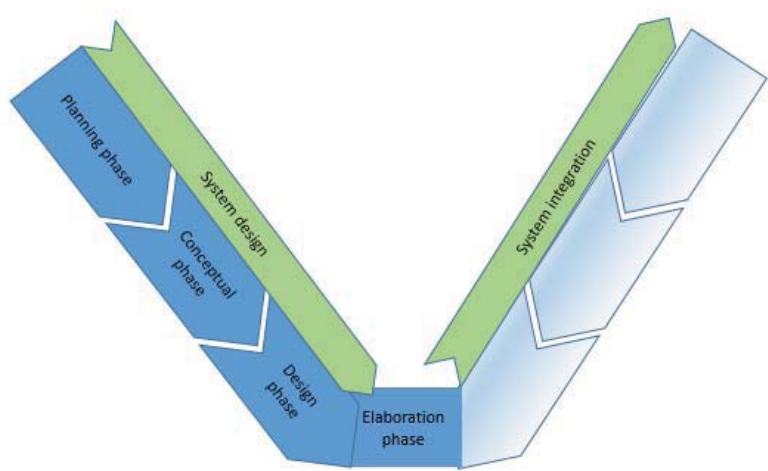

Figure 1. Combination of four phases and V-model ([14] page $44]$

Adding the input from the ISO 26262 leads to figure 2

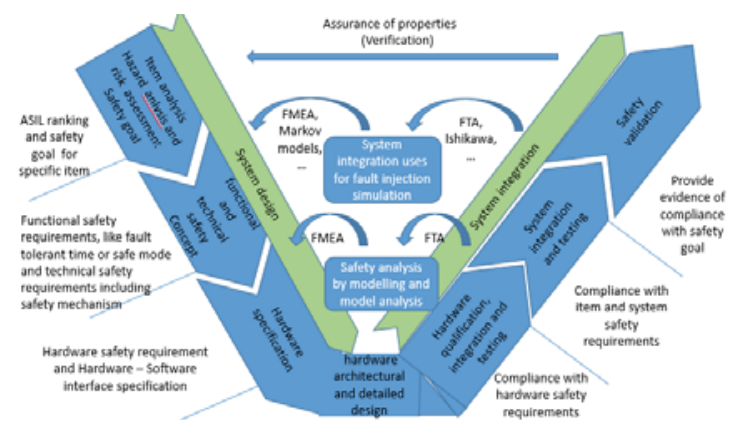

Figure 2. Adding input from ISO26262 ([14] page 45]

As the focus of the new methodology is the left branch of the $\mathrm{V}$-model during the design phase is considered only. 
Therefore, the following new methodology is proposed

"1. Selection of a hardware-orientated safetyrelated function in the automotive sector.

Not each function can be selected. It has to be a function, whose safety dependence is carried out purely by hardware. Safety functions which are performed by software are of course hardware-based, but not concerned within the focus of this work.

2. Performing the Functional Safety activities in accordance to ISO 26262

According to the ISO 26262 each Functional Safety analysis has to be started with the following steps:

- Item analysis;

- Hazard and risk analysis;

- Risk Classification;

- Deriving the safety goals;

- Setting up a functional and technical safety concept. (compare [3] part 3)

3. Using an aging failure mode and effect analysis (FMEA) to find aging relevant hardware components which safety-related functions

Implementation of an "aging" FMEA is subjected with the aim of detecting aging dependent critical hardware components that can cause a potential hazard to the safety goal or false failure diagnosis.

\section{Development of a VHDL-AMS model with the} capability to simulate aging behaviors

Development of component models, for the simulation of the aging behavior of the technical safety concept.

The data source for the aging input for the models can be from

a) data sheets or scientific papers;

b) field data (proven in use) (compare [3] part 8 page 35)

c) own experiments.

\section{Simulation with VHDL-AMS}

The simulation is applied over the range of evidence. The aim to make sure, that safety goals are not violated, and the diagnosis intervals are correct. Simulation is performed over the lifetime, with the aim to determine the robustness of the chosen concept.

\section{Evaluation of the results}

The advantage of a simulation is also to look on certain values to see how big or how small the safety margins are." ( [14] page 46):

\section{Application of NeW Methodology ON A SENSOR OF THE EGAS FUNCTION}

\section{A. Selection of a suitable safety-related function}

In order to prove the new methodology, it is applied on the throttle valve sensor of the EGAS Function (compare [8]). "The EGAS is the function inside a direct injection gasoline engine which converts the driver request into a torque at the tires, by controlling the air - gasoline mixture at the intake of the cylinder. By the proportion of the air gasoline mixture the optimum between power output and best combustion is influenced. The wish of the driver is measured with the accelerator pedal module, which measures the angle of the pedal. This can be done by potentiometers or by hall-sensors. This input signal together with other sensors (e.g. knock sensor) is the basis for the engine control unit to control the electronic throttle valve. The position of the valve is read back by potentiometers in a voltage divider circuit." ([14], page 48)

The main $\mathrm{E} / \mathrm{E}$ components are the acceleration pedal module, the engine control unit, and the throttle valve.

The electrical equivalent circuit for the positioning sensor of the throttle valve is a simple voltage divider and is shown in figure 3 .

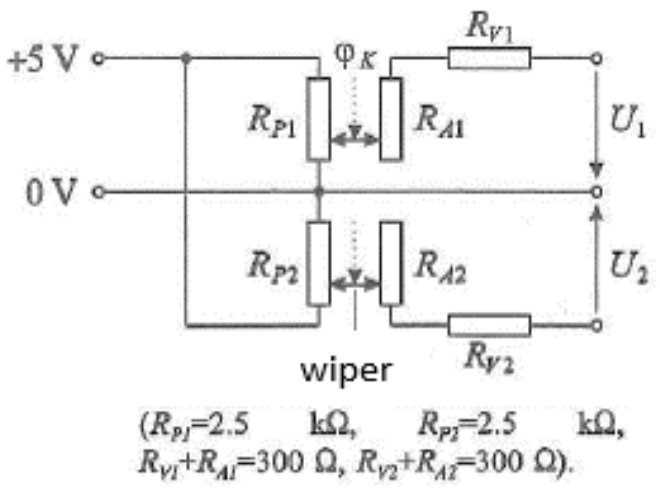

Figure 3. electrical equivalent circuit of a throttle valve ([10], page 15)

There are two wipers and two voltage dividers to achieve a redundancy. Different independent polarity for the voltage supply is used to plausible the signals form the two voltage dividers.

\section{B. Performing functional safety activities in accordance with ISO 26262}

A working group consistent of the automotive industry with representatives from Audi AG, BMW AG, Daimler AG, Porsche AG and VW AG published together their common requirements for a "standardized E-GAS Monitoring concept for gasoline and diesel engine control units" (compare 
[8]). There it is specified that the highest ASIL ranking of the EGAS function is "ASIL B" and the corresponding safety goal is "prevention of unintended acceleration" ([8], page 9). Further a "plausibility check can be applied to the actuator signals (e.g. throttle position) after capturing the signal" ([8], page 10) and for the ECU a 3 level monitoring safety concept is proposed ([8], page 13).

\section{Finding aging relevant hardware by using an Aging FMEA}

A failure mode and effect analysis is a proactive method to analyze and optimize a unit, system or a process in a systematic way, how it reacts to failures. Additionally, it provides a documentation to prove that a safety analysis has been performed (compare [7], page 7).

Different kinds of FMEAs exist, for example design, process, software, logistic FMEA. (compare [11], page 13). This means there can be a focus where the FMEA is concentrated on. Following this approach here the proposal is to use the method FMEA with the focus aging. The approach is to look on each component and evaluate it, if there can be a functional impact by an aging behavior of the component.

For demonstration purpose the potentiometer of the throttle valve is identified as aging relevant.

\section{Developing VHDL-AMS Model}

In order to develop a VHDL AMS Model which includes aging - there has to be a mathematical formula which describes the aging behavior.

In order to demonstrate the method a simplification was done. Instead of a potentiometer a simple voltage divider with a fixed ratio was used. The formula for resistor was taken out of the paper "The Aging Behavior of Commercial Thick-Film Resistors" [12] and the variables where estimated with the curve fitting Toolbox from MATLAB[13].

It is also possible to do own experiments or use field data to develop a mathematical formula.

\section{E. Simulation and Evaluation of result}

By simulating the equivalent circuit as voltage dividers with aging effects, no influence is shown. The reason for this is, that the simulation models for the single resistor were completely identically. So extreme scenarios, that only one resistor is aging, were investigated. In the following one example/scenario is explained.

In the described scenario $1 b$ (see figure 4), only one resistor at the positive voltage source circuit has the aging function according to an e-function corresponding the paper of Sinnadurai and Wilson (compare [12]). To simulate the two voltage dividers (positive and negative voltage supply) two circuits are simulated - the simulator ANSYS Simplorer version 16.2 is used. The model of the circuits is shown in figure 4 .

\section{positive voltage source}

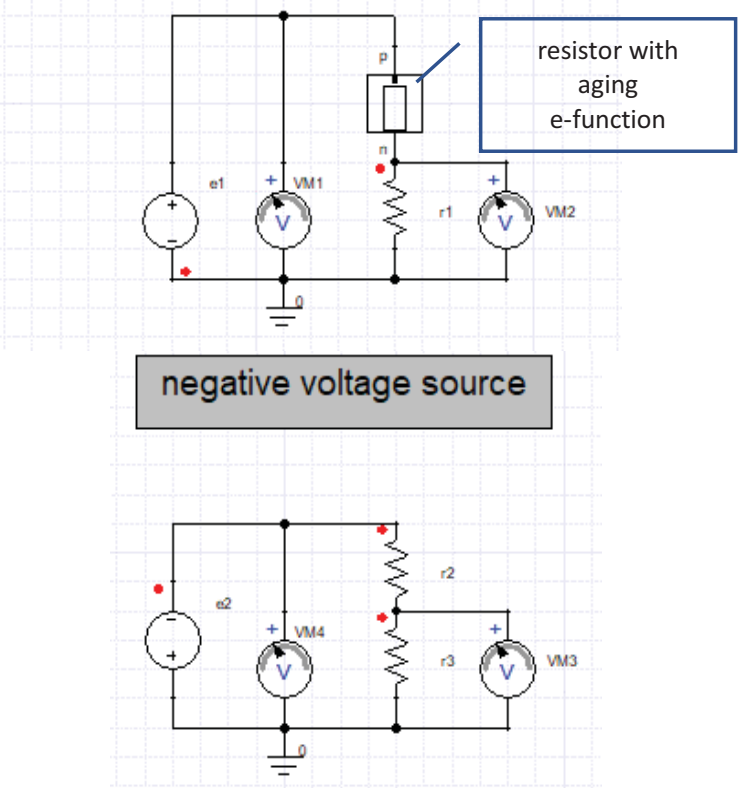

Figure 4. Simplorer model for simplified voltage divider (throttle position) scenario with one resistor applied by e-function aging ([14] page 86 )

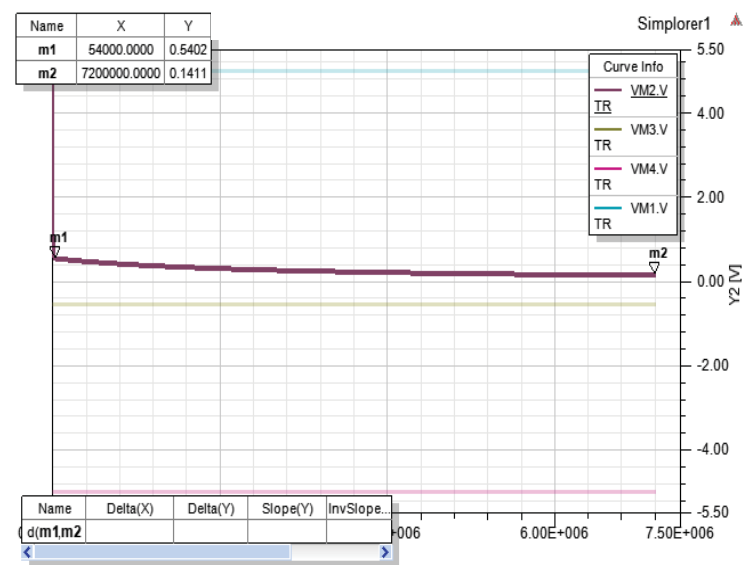

Figure 5. result of simulation scenario 1b ([14], page 88)

Figure 5 shows the voltage on the non aging resistor in the positive voltage circuit (VM2) in a diagram. With the values from the marker (see table in the left top corner of figure 5) - a change in the voltage is seen from $0,5402 \mathrm{~V}$ to $0,1411 \mathrm{~V}$ from simulation start to simulation end $(2000 \mathrm{~h})$ - which means the voltages by $73 \%$. This value changes is significat, so that this is an indicator that further design activities are needed. The internal tolerance limits are know how of the different manufacturer. 


\section{CONCLUSION}

"Summarizing it is shown that the described methodology is an additional contribution to a structural approach of improving the design reaching the safety goals and optimizing the diagnostic mechanism in an early design phase by aging simulation." ([14], page 90]

Due to this benefit it is proposed to consider the aspect aging in the next edition of the ISO 26262, especially in the part 5 for hardware design.

It has to be mentioned that this methodology is a supplement to existing methods and with the current data base it cannot substitute the actual environmental validation. The main limitations are the available aging information for the components with safety influence. This could be overcome by requesting the models in the ISO 26262. (compare [14] page 90)

"As a further research field it is seen how aging mechanism influence electronic components and systems. Also, the methodology can further be used to develop or investigate safety reaction, like diagnostics.” ([14] page 90)

\section{REFERENCES}

[1] VDI 2221:1993 Verein deutscher Ingenieure (VDI), VDI 2221 Methodik zum Entwickeln und Konstruieren technischer Systeme und Produkte Berlin, Beuth Verlag 1993

[2] VDI 2206:2004, Verein deutscher Ingenieure (VDI), VDI 2206 Design methodology for mechatronic systems, Berlin, Beuth Verlag, 2004

[3] ISO 26262:2011, ISO 26262, road vehicles - functional safety, part 1 to 10 , Genf, ISO, 2011

[4] Brünglinghaus, C. http://www.springerprofessional.de/elektronik-und-softwarebeherrschen-innovationen-im-auto/5097588.html accessed 13.08.2018

[5] ISO 16750-4:2010, Road vehicles- Environmental conditions and testing for electrical and electronic equipment - Part4: Climatic loads, 2010

[6] Reif, K. (2014), Automobilelektronik, $5^{\text {th }}$ edition, Wiesbaden, Springer-Verlag, 2014

[7] DIN EN 60812, Failure mode and effects analysis - draft, Beuth-Verlag, Berlin, 2017

[8] EGAS (2013) Standardized E-Gas Monitoring Concept for Gasoline and Diesel Engine Control Units, Version 5.5, Specification of the E-GAS Working group (Audi, BMW, Daimler, Porsche, VW) 2013, https://www.iav.com/sites/default/files/attachments/seite/akegas-v5-5-en-130705.pdf accessed 28.02.2016

[9] Hofmann, G, Georgiev, V (2016)., Using VHDL-AMS to simulate aging behavior of a resistor, 2016 International Conference on Applied Electronics (AE), Pilsen, 2016, pp. 89-92

[10] Pfeufer, T. (1999), Modelgestützte Fehlererkennung und Diagnose am Beispiel eines Fahrzeugaktuators; in Fortschritts-Berichte VDI Reihe $8 \mathrm{Meß}-$, Steuerungs- und Regelungstechnik, Nr 749, Düsseldorf, VDI-Verlag, 1999

[11] Werdich, M. (editor), (2012) FMEA - Einführung und Moderation, 2. Auflage, Springer + Teubner Verlag, Wiesbaden, 2012

[12] Sinnadurai, N.; Wilson, K. (1982), The Aging Behavior of Commercial Thick-Film Resistors, Components, Hybrids, and Manufacturing Technology, IEEE Transactions on, IEEE, 1982

[13] https://de.mathworks.com/products/matlab.html accessed on 03.03.2019

[14] Hofmann, G, dissertation New design methodology - Using VHDL-AMS models to consider aging effects in automotive mechatronic circuits for safety relevant functions, university of West Bohemia, 2018

\footnotetext{
${ }^{\mathrm{i}}$ This paper is a summary of the dissertation [14] with the same title and this dissertation is the main source of information
} 